\title{
Tandem Mass Spectrometric Investigation of Acylpolyamines of Spider Venoms and Their ${ }^{15} \mathrm{~N}$-Labeled Derivatives
}

\author{
Manuel Tzouros, Nikolay Manov, Stefan Bienz, and Laurent Bigler \\ Institute of Organic Chemistry, University of Zurich, Zurich, Switzerland
}

\begin{abstract}
The fragmentation mechanism of the acylpentamine toxins 1-4 found in the venom of the spider Agelenopsis aperta has been investigated in detail. To identify the origin of the two doublets of unexpected fragment ions at $\mathrm{m} / \mathrm{z} 129 / 112$ and $\mathrm{m} / \mathrm{z} 115 / 98$, three synthetic ${ }^{15} \mathrm{~N}$-labeled analogs 5-7 have been prepared and subjected to CID fragmentation on a triple quadrupole mass spectrometer. It appears that the unexpected doublet of fragment ions arises from an internal portion of the polyamine backbone after either a transaminative Zip reaction or a sequential fragmentation of the quasi-molecular ion. The second option has been proven by in-source CID experiments. The detailed knowledge of acylpentamine fragmentation mechanisms is essential for the correct characterization of isomeric compounds, particularly for coeluting compounds within complex mixtures such as spider venoms. (J Am Soc Mass Spectrom 2004, 15, 1636-1643) (c) 2004 American Society for Mass Spectrometry
\end{abstract}

$\mathrm{P}$ olyamines and polyamine-containing alkaloids are widely found throughout the plant and animal kingdom [1, 2]. Over the past decades, spider venoms, particularly their fractions containing acylpolyamine derivatives $[1,3,4]$, have increasingly attracted the attention of scientists. With the advent of more sensitive and selective analytical methods-in particular of the modern mass spectrometric approaches-not only the major but also minor constituents of such trace toxin sources became amenable to detection and structural elucidation. Only recently have we demonstrated with the reinvestigation of the venom of the spider Agelenopsis aperta (Agelenidae) that this toxin cocktail contains in addition to the previously found 11 major acylpolyamines, 27 further minor components that evaded earlier investigations $[5,6]$.

The detection and structural elucidation of these spider toxins relied particularly on the on-line coupled high-performance liquid chromatography, UV diode array detection, and atmospheric-pressure chemical ionization mass spectrometry [HPLCUV(DAD)-APCI-MS and -MS/MS]. This arrangement allowed the efficient separation and investigation of the several venom components, making most economical use of the precious sample material. The structural elucidations, however, would not have been possible if a profound knowledge of the frag-

Published online September 22, 2004

Address reprint requests to Dr. L. Bigler, Institute of Organic Chemistry, University of Zurich, Winterthurstrasse 190, CH-8057 Zurich, Switzerland. E-mail: lbigler@oci.unizh.ch mentation behavior of the parent class of compounds had not been available, especially in the case of coeluting isomers. While most of the toxins of $A$. aperta were disclosed on the basis of pure "MS investigations" by comparison of fragmentation patterns observed and explained during previous investigations, five of them were only revealed by their comparison with synthetic material [6]. But also in these cases, their identity could only be revealed because of the specific fragmentation behavior of several structural isomers that could only be studied, however, with the pure sample compounds. On the whole, the knowledge of the fragmentation patterns of polyamines and also the deeper understanding of the respective fragmentation mechanisms proved an important prerequisite to allow efficient and definitive identification and characterization of known and new polyamine derivatives by MS methods.

In the course of our investigation of the venom of A. aperta, we have studied the fragmentation behavior of acylpolyamines extensively and have also discussed possible fragmentation paths and mechanisms [5]. For some groups of isomeric acylpentamines, however, inconsistencies with regard to expected fragmentation patterns surfaced. Concentrating solely on the proposed fragmentation reaction for the "loss of the terminal diaminopolymethylene unit", the occurrence of fragments of the types a and $\mathbf{b}$-formed upon collision induced dissociation (CID) by intramolecular nucleophilic substitutions $\left(\mathrm{S}_{\mathrm{N}} \mathrm{i}\right)$ according to Scheme 1 -were thought to be indicative for the identification of the terminal diamine portions 

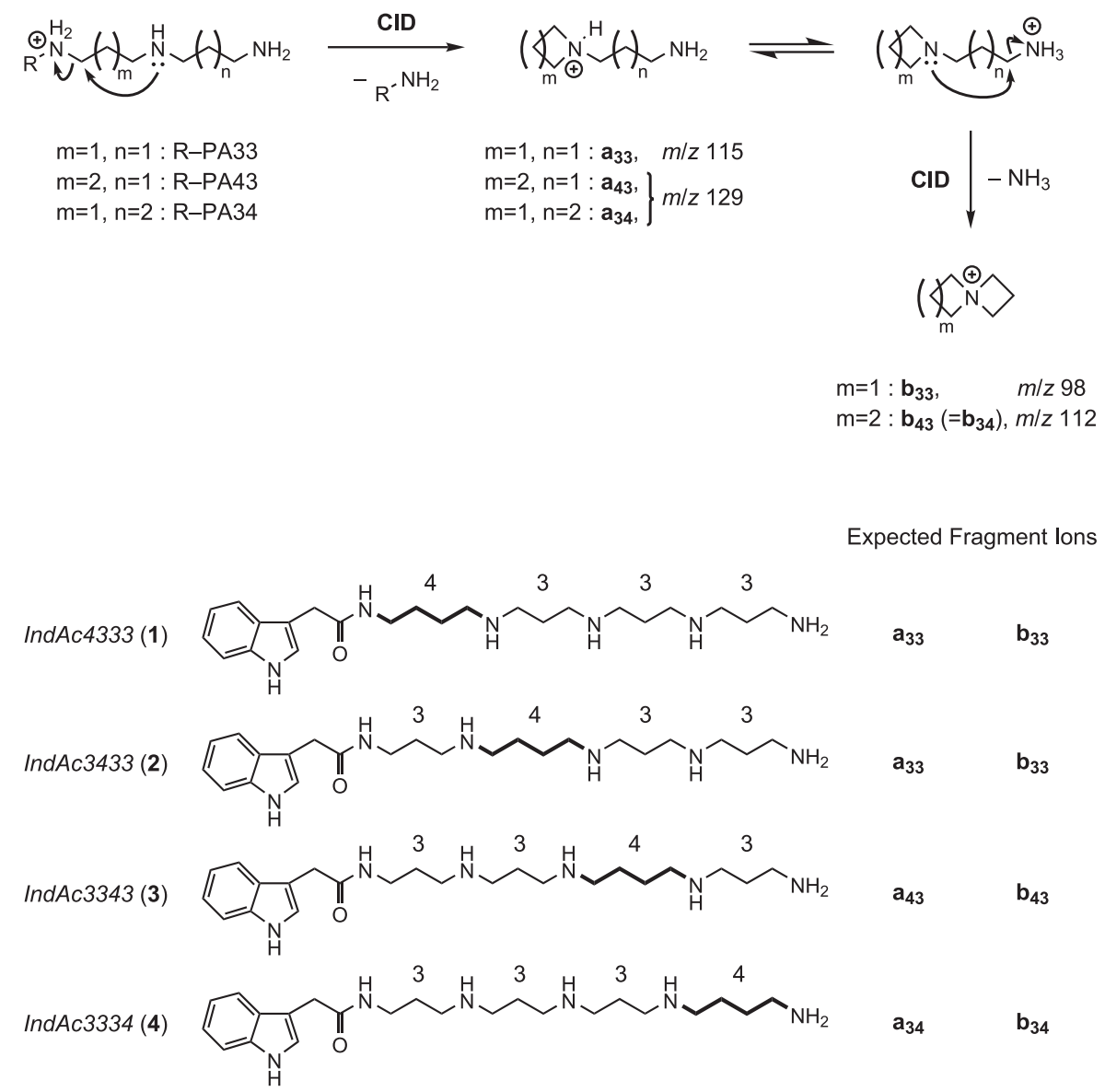

Scheme 1. Mass spectral fragmentation of the terminal part of the $[\mathrm{M}+\mathrm{H}]^{+}$ions of the IndAc pentamine derivatives $\mathbf{1 - 4}$.

of the toxins. However, this is only true with reservation. For instance, the toxins IndAc4333 [the expression IndAc4333 stands for a linear pentamine possessing $4,3,3$, and 3 methylene units between the several $\mathrm{N}$-atoms, whereby the IndAc group is located at the $\mathrm{N}$-atom which is followed by the tetramethylene unit. Ind $A c$ is the abbreviation for $1 H$-indole-3-acetyl] (1) and IndAc3433 (2) would be expected to give rise to fragments $\mathbf{a}_{33}$ [the subscripts denote the number of methylene groups contained in the two chains or rings] $(\mathrm{m} / \mathrm{z} 115)$ and $\mathbf{b}_{33}(\mathrm{~m} / \mathrm{z} 98)$ and not to fragments $\mathbf{a}_{43} / \mathbf{a}_{34}(\mathrm{~m} / \mathrm{z} 129)$ and $\mathbf{b}_{43}(\mathrm{~m} / \mathrm{z} 112)$, while isomeric IndAc3343 (3) and IndAc3334 (4) should produce fragments $\mathbf{a}_{43} / \mathbf{a}_{34}$ and $\mathbf{b}_{43}$ instead, and none of the fragments $\mathbf{a}_{33}$ and $\mathbf{b}_{33}$.

In reality, however, the CID spectra derived from the two quasi-molecular ions of synthetic IndAc4333 and IndAc3433 also showed signals at $\mathrm{m} / \mathrm{z} 129$ and 112, which should be indicative for a terminal PA34 or PA43 unit; [PA34 stands for a linear triamine with 3 and 4 methylene units (in this succession) between the terminal and internal $\mathrm{N}$-atoms [2]. The nomenclature is also used for more extended polyamines.] In the case of IndAc3334 (but not of IndAc3343!), signals at $\mathrm{m} / \mathrm{z} 115$ and 98 were additionally found, which would imply a PA33 end-portion for the molecule (Figure 1). The feature of exhibiting unexpected signals of such types is not specific to pentamines bearing the IndAc moiety; it is also characteristic for other pentamine derivatives that bear, e.g., a 4-hydroxybenzoic or a 2,5-dihydroxybenzoic group in place of IndAc (spectra not shown here).

As an explanation for the peculiar MS behavior of the acylpentamines, we have offered two plausible reaction paths involving an acid promoted transamidative (A) or transaminative (B) Zip reaction [7], as outlined in Scheme 2 in the case of IndAc3334 (4). For both transformations, precedence is available in the MS literature: Transamidation reactions were discussed with respect to the MS behavior of 4-hydroxycinnamoyl spermidines [8] as well as $N, N^{\prime}$-bis(4-hydroxycinnamoyl) spermidines [9], and transamination reactions were considered for polypropylenamine dendrimers [10] and for monoimidazole polyamine conjugates [11]. The presence of the signal doublet at $\mathrm{m} / \mathrm{z} 129 / 112$ in the CID of IndAc4333 would rather support the transamidative Pathway A while the lack of signals at $\mathrm{m} / \mathrm{z}$ 115/98 for IndAc3343 would suggest Pathway B proceeding through transaminations.

To distinguish between these two paths, we have 
(a)

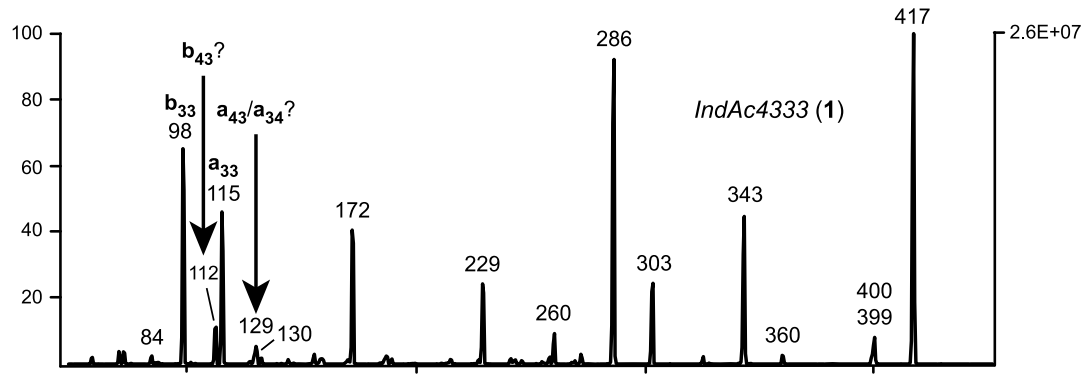

(b)

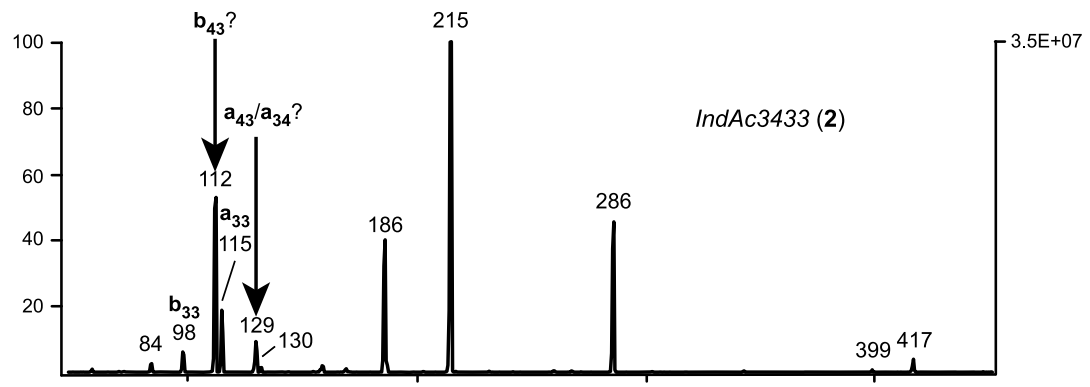

(c)
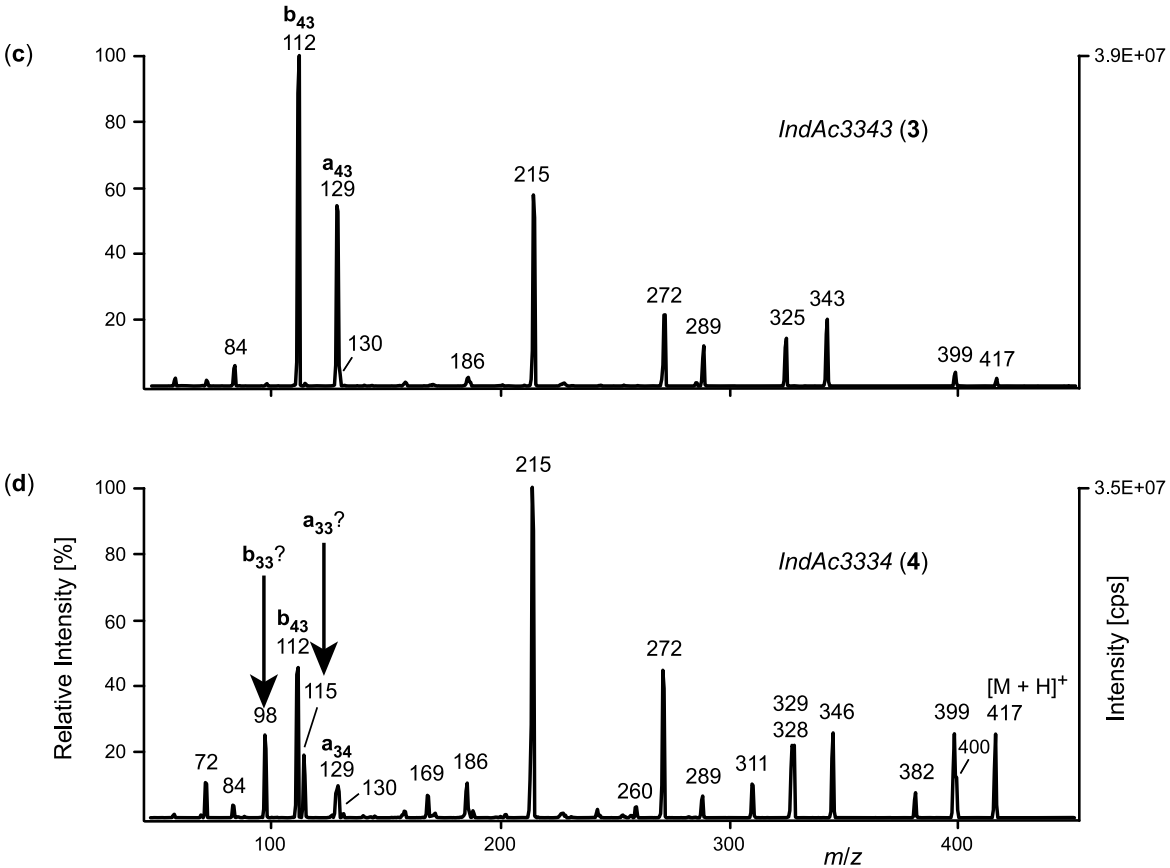

Figure 1. ESI-MS/MS of the $[\mathrm{M}+\mathrm{H}]^{+}$ion at $m / z 417$ of (a) (1), (b) (2), (c) (3), and (d) (4). Unexpected fragment ions are indicated by an arrow.

synthesized the three ${ }^{15} \mathrm{~N}$-containing IndAc3334 derivatives $\operatorname{Ind} A c^{15} \mathrm{~N} 3334$ (5), $\operatorname{IndAc} 3^{15} \mathrm{~N} 334$ (6), and IndAc $33^{15} \mathrm{~N} 34$ (7) [12] (Figure 2). The three labeled isomers were then analyzed by ESI-MS/MS, analogously to the unlabeled parent compound. The results of this MS investigation are presented below.

\section{Experimental}

\section{Material}

All ${ }^{15} \mathrm{~N}$-labeled acylpolyamine samples used were synthesized in our laboratory [12]. For ESI-MS, all com- pounds were dissolved in a 1:1 mixture of HPLC grade $\mathrm{MeOH}$ (Scharlau, Barcelona, Spain) and $\mathrm{H}_{2} \mathrm{O}$ (purified with a Milli- $Q_{\text {RG }}$ apparatus, Millipore, Milford, MA) at a concentration of $50 \mathrm{nmol} \mathrm{ml} l^{-1}$.

\section{Mass Spectrometry}

ESI tandem mass spectra were recorded with a TSQ 700 triple quadrupole mass spectrometer (Finnigan MAT, San Jose, CA) equipped with a combined Atmospheric Pressure Ion (API) source. Samples were continuously introduced into the source with a Syringe Pump 22 


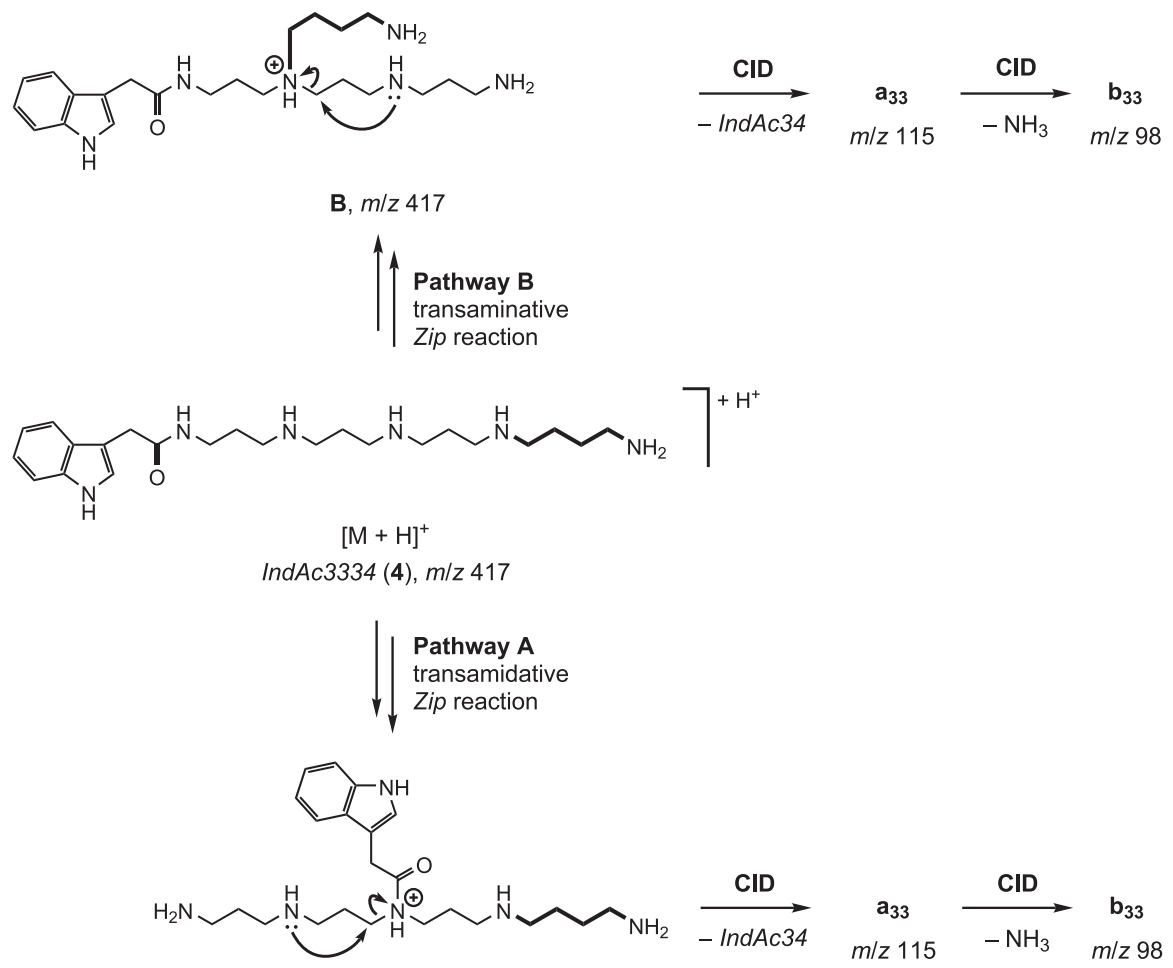

A, $m / z 417$

Scheme 2. Possible gas phase rearrangements of the $[\mathrm{M}+\mathrm{H}]^{+}$ions of IndAc3334 (4).

(Harvard Apparatus, Holliston, MA) at a flow rate of 5 $\mu \mathrm{l} \mathrm{min}^{-1}$. The ESI operating conditions in positive mode were: capillary voltage $4500 \mathrm{~V}$; heated capillary temperature $210{ }^{\circ} \mathrm{C}$; sheath gas $\mathrm{N}_{2}$ with an inlet pressure of 30 psi; electron multiplier: $1000 \mathrm{~V}$; conversion dynode: $-15 \mathrm{kV}$; resolution: $0.7 \mathrm{u}$ at half peak height; scan rate: $900 \mathrm{Da} \mathrm{s}^{-1} ; 16$ scans were averaged. For the CID experiments, Ar was used as the collision gas with a relative pressure of 2.5-3.3 mTorr. The collisioninduced dissociation offset (Coff) was set to $-27 \mathrm{~V}$ and the electron multiplier was set to $1500 \mathrm{~V}$. In-source CID spectrum was obtained by setting the octopole voltage at $-3 \mathrm{~V}$, and fragment ions were further subjected to CID by setting the Coff voltage at $-22 \mathrm{~V}$.

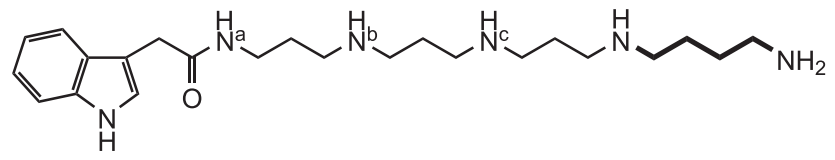

$$
\begin{aligned}
& \mathrm{N}^{\mathrm{a}}={ }^{15} \mathrm{~N}: \operatorname{IndA} A c^{15} \mathrm{~N} 3334(5),[\mathrm{M}+\mathrm{H}]^{+} \mathrm{m} / \mathrm{z} 418 \\
& \mathrm{~N}^{\mathrm{b}}={ }^{15} \mathrm{~N}: \operatorname{Ind} A c 3^{15} \mathrm{~N} 334(\mathbf{6}) \\
& \mathrm{N}^{\mathrm{c}}={ }^{15} \mathrm{~N}: \operatorname{Ind} A c 33^{15} \mathrm{~N} 34(\mathbf{7})
\end{aligned}
$$

Figure 2. Investigated ${ }^{15} \mathrm{~N}$-labeled IndAc3334 derivatives 5-7.

\section{Results and Discussion}

The ESI-MS/MS of the three labeled Compounds 5-7 and of the unlabeled parent Compound 4-all obtained under the same conditions from the respective $[\mathrm{M}+\mathrm{H}]^{+}$parent ions-are shown in Figure 3 . Concentrating on the signals of type $\mathbf{a}$ and $\mathbf{b}$, it is readily recognized that the signals for the respective fragment ions $\mathbf{a}_{34} / \mathbf{b}_{43}$ [the fragment ion at $\mathrm{m} / \mathrm{z}$ 130 has been previously described as 3-methylene$3 \mathrm{H}$-indolium $\left(\mathrm{C}_{9} \mathrm{H}_{8} \mathrm{~N}^{+}\right)$[5] and is not considered as labeled $\left.{ }^{15} \mathrm{~N}-\mathbf{a}_{43} / \mathbf{a}_{34}\right]$ at $m / z 129 / 112$ remained unaffected for all the ${ }^{15} \mathrm{~N}$-containing compounds. However, the signals for the $\mathbf{a}_{33} / \mathbf{b}_{33}$ ions were recorded only for $I n d A c^{15} N 3334$ (5) and $I n d A c 3^{15} \mathrm{~N} 334$ (6) at $\mathrm{m} / \mathrm{z}$ $115 / 98$ while they were found at masses higher by 1 Da $\left(\mathrm{m} / \mathrm{z}\right.$ 116/99) for $\operatorname{IndAc} 33^{15} \mathrm{~N} 34$ (7) and consequently designated as ${ }^{15} \mathrm{~N}-\mathbf{a}_{33}$ and ${ }^{15} \mathrm{~N}-\mathbf{b}_{33}$, respectively.

These results unambigously show that the central portion of the polyamine moiety (as indicated by the surrounded part of the compound in Figure 4) is incorporated in the fragments $\mathbf{a}_{33} / \mathbf{b}_{33}$ deriving from Compound 4 . Therefore, the transamidative Pathway A for the formation of these fragments is certainly not operative and can be excluded. Otherwise, doublets for labeled fragments $\mathbf{a}_{33} / \mathbf{b}_{33}$ at $m / z 116 / 98$ or $116 / 99$ should have been found for Isomers 5 and $\mathbf{6}$, respectively. On the other hand, the doublet of signals at $m / z$ 116/99 for 
(a)

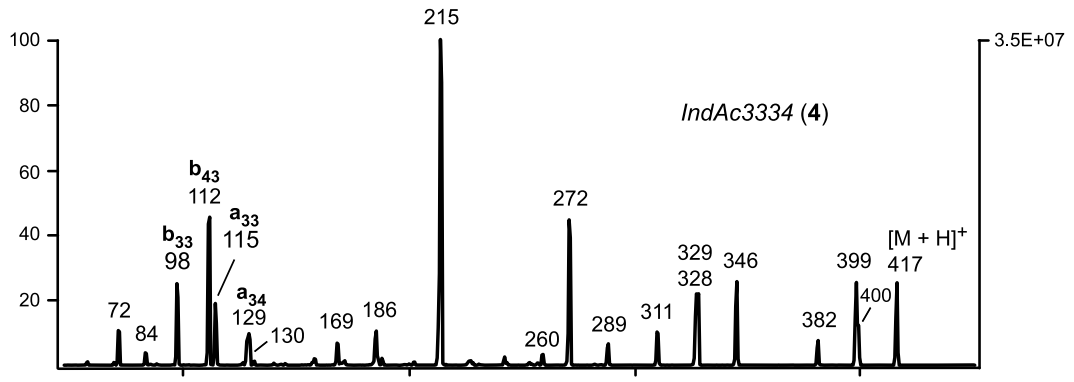

(b)

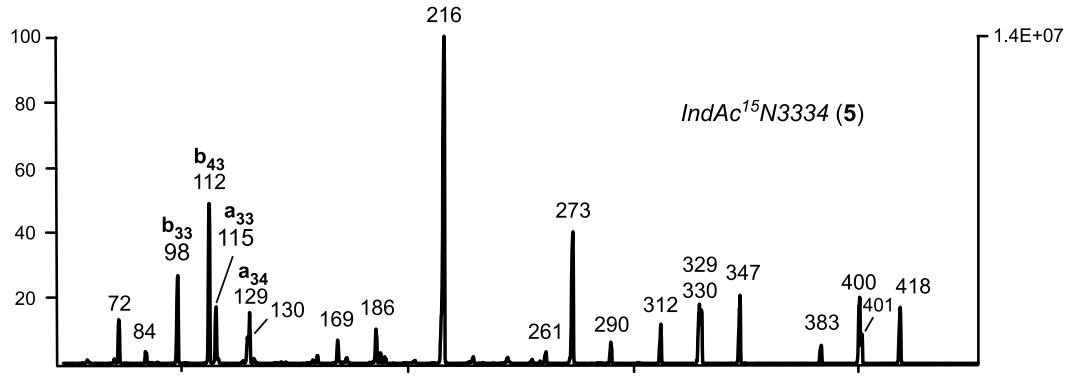

(c)

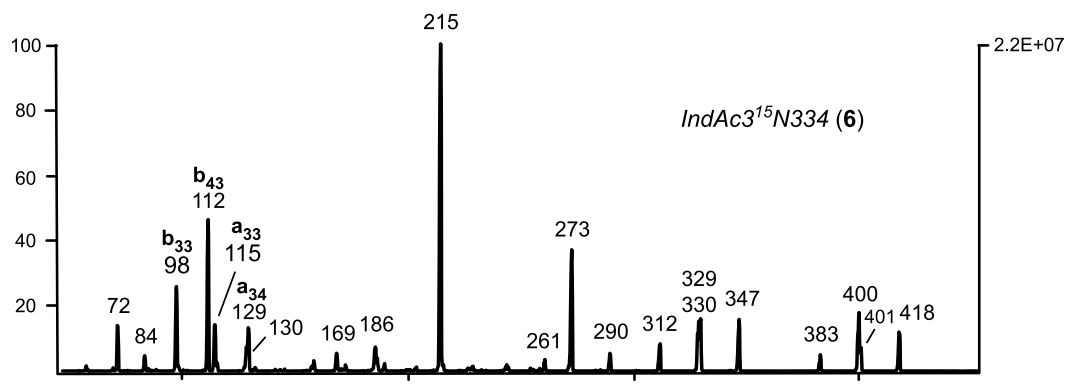

(d)

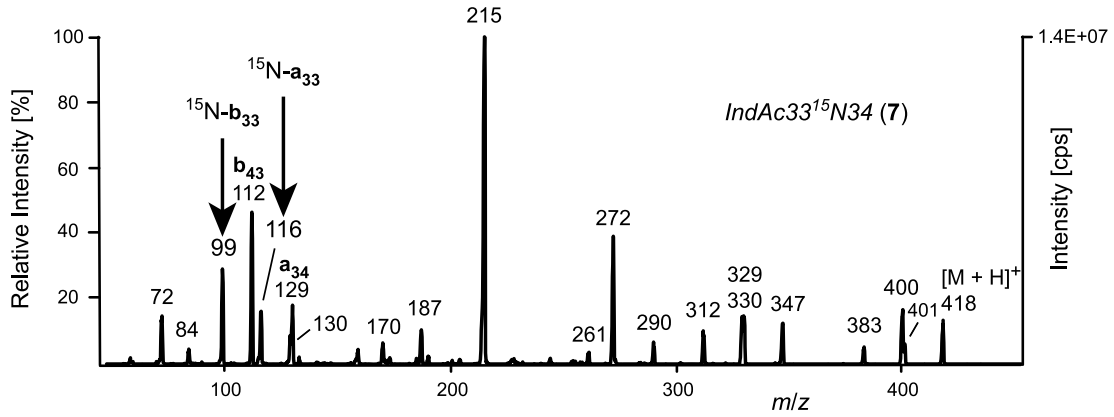

Figure 3. ESI-MS/MS of the $[\mathrm{M}+\mathrm{H}]^{+}$ions at $\mathrm{m} / z 417$ of (a) $\operatorname{IndAc} 4333$ (4), and at $\mathrm{m} / \mathrm{z} 418$ of labeled (b) IndAc ${ }^{15} N 3334$ (5), (c) IndAc $3{ }^{15} N 334$ (6), and (d) IndAc33 ${ }^{15} N 34$ (7). ${ }^{15} \mathrm{~N}$-label containing a and b fragment ions are indicated by an arrow.

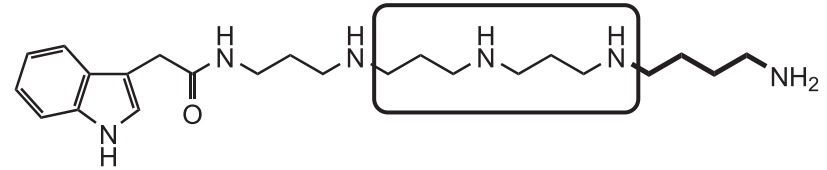

Figure 4. Portion of the polyamine backbone of IndAc3334 responsible for the formation of the fragment ions $\mathbf{a}_{33} / \mathbf{b}_{33}$ at $\mathrm{m} / \mathrm{z} 115 / 98$. the ${ }^{15} \mathrm{~N}$-containing fragments $\mathbf{a}_{33} / \mathbf{b}_{33}$ deriving from Isomer $\mathbf{7}$ is supportive for the transaminative Pathway B.

An alternative fragmentation path that was not seriously considered initially, but is also consistent with the experimental data, is the sequential fragmentation of acylpolyamines involving loss of a terminal aminoalkyl group in a first stage, forming, e.g., for IndAc3334 the ion $[\operatorname{IndAc} 333+\mathrm{H}]^{+}$. Such a secondary parent ion could subsequently be fragmented according to the general 

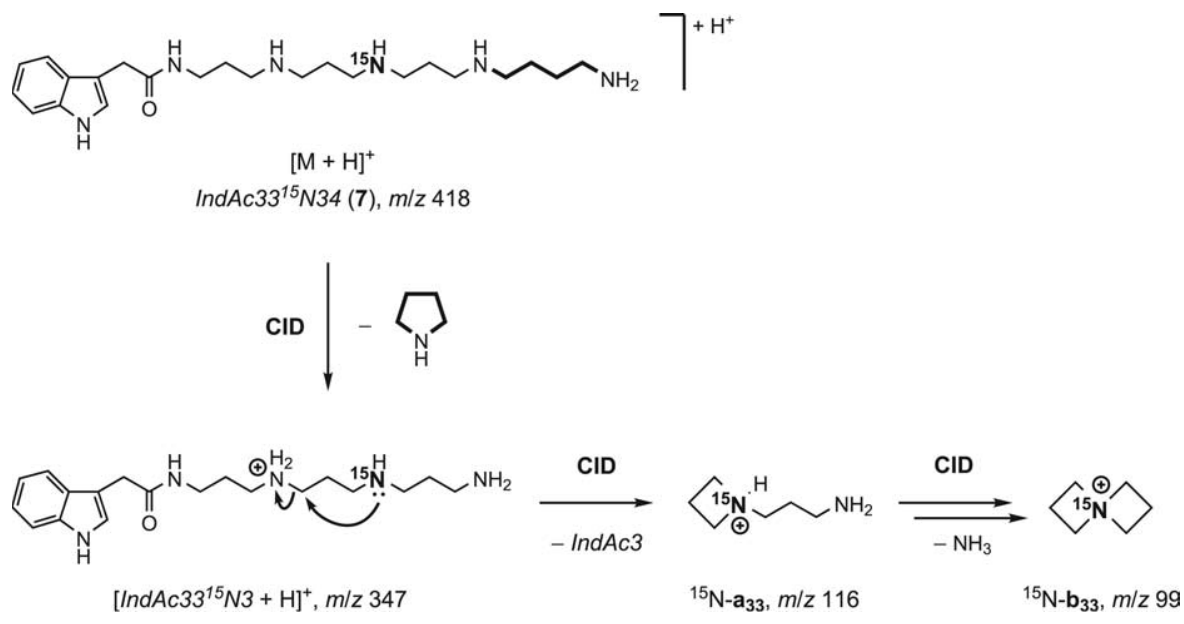

Scheme 3. Sequential mass spectral fragmentation of the $[\mathrm{M}+\mathrm{H}]^{+}$ions of $\operatorname{IndAc} 33^{15} \mathrm{~N} 34$ (7).

rules (Scheme 3). This would lead to fragments $\mathbf{a}_{33}$ and $\mathbf{b}_{33}$ that would consist of the same portions of the parent molecule as those explained with the transamination/ fragmentation pathway. However, if IndAc3334 dissociates by the usual $\mathrm{S}_{\mathrm{N}} \mathrm{i}$ reaction by attack of the terminal amino group at the $\mathrm{CH}_{2}$ group in $\alpha$-position to the protonated secondary $\mathrm{N}$-atom, protonated pyrrolidine (see fragment ions at $\mathrm{m} / \mathrm{z} 72$ in Figure 3) and neutral IndAc333 is formed. Thus the observed [IndAc $33^{15} \mathrm{~N} 3+$ $\mathrm{H}]^{+}$at $\mathrm{m} / \mathrm{z} 347$ from $\operatorname{IndAc} 33^{15} \mathrm{~N} 34$ (7) cannot directly derive from this $S_{N} i$ reaction. In order to retain the charge on the IndAc-end, a proton transfer process has to be operative. It is possible that a proton-bound complex is formed during fragmentation, where the IndAc333-unit and pyrrolidine compete for the $\mathrm{H}^{+}$. This mechanism was postulated to explain the formation of the $b_{n} / y_{n}$ ion series in the case of peptide fragmentation [13].

Support for the sequential fragmentation of $4-7$ is found with the signals at $\mathrm{m} / \mathrm{z} 346$ and 347 , corresponding to the proposed ions $[\operatorname{IndAc333}+\mathrm{H}]^{+}$ (Figure 3). That these ions are formed rather easily is also demonstrated with the in-source CID experiment, which involves very mild energy transfer and thus very mild fragmentation conditions, performed with IndAc33 ${ }^{15} \mathrm{~N} 34$ (7) (Figure 5). By in-source CID, almost exclusively, the respective ions $\left[\operatorname{Ind} A c 33^{15} \mathrm{~N} 3\right.$ $+\mathrm{H}^{+}$registered at $m / z 347$ were formed. These ions, selected by the first quadrupole and subjected to the usual CID by collision with Ar in the collision cell,

(a)

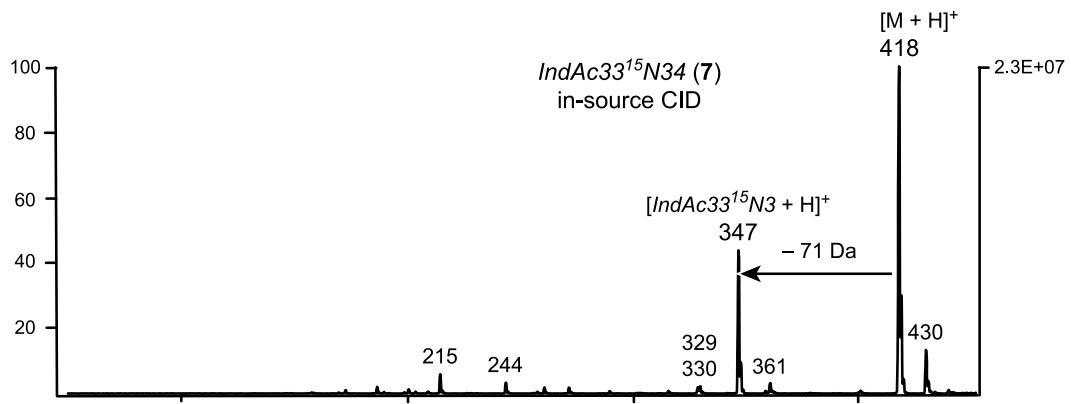

(b)

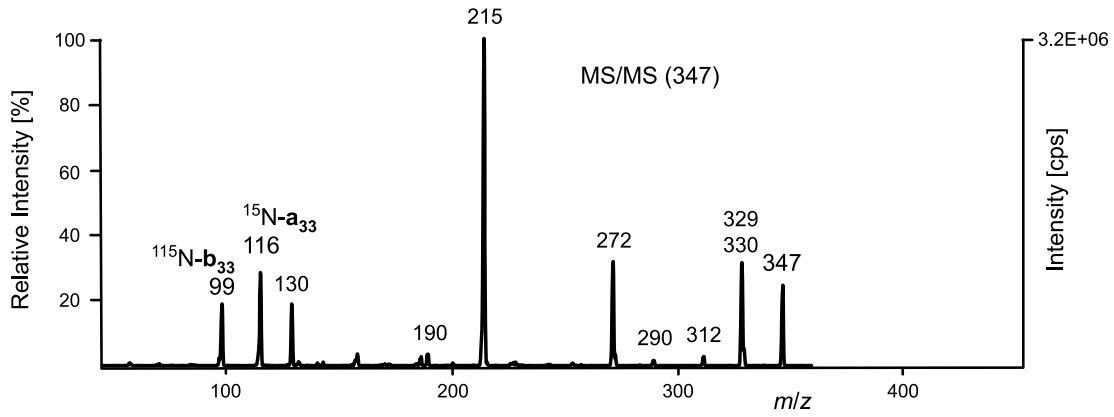

Figure 5. In-source CID of the $[\mathrm{M}+\mathrm{H}]^{+}$ion at $\mathrm{m} / \mathrm{z} 418$ of labeled (a) $\operatorname{IndAc} 33^{15} \mathrm{~N} 34$ (7), and (b) MS/MS of the secondary parent $\left[\operatorname{IndAc} 33^{15} \mathrm{~N} 3+\mathrm{H}\right]^{+}$ion at $\mathrm{m} / z 347$. 
gave rise to a spectrum that exhibited, not surprisingly, signals at $\mathrm{m} / \mathrm{z} 116$ and 99 for the ${ }^{15} \mathrm{~N}$-labeled fragments of type $\mathbf{a}_{33}$ and $\mathbf{b}_{33}$.

Based on the experimental data acquired by the above experiments, both mechanisms-decomposition of $[\text { IndAc3334 }+\mathrm{H}]^{+}$through transamination/fragmentation (Pathway B) or by sequential fragmentation via intermediary [IndAc333 $+\mathrm{H}]^{+}$ions-are feasible to explain to the formation of fragments $\mathbf{a}_{34} / \mathbf{b}_{34}$ detected at $m / z 129 / 115$ in the ESI-MS/MS of Compound 4. The fact that $[\operatorname{IndAc333}+\mathrm{H}]^{+}$is formed quite easily by in-source CID and that this ion is also detected in the regular CID spectra talks in favor of the sequential fragmentation path.

The behaviors of IndAc3433 (2) and IndAc3343 (3) are consistent with the two fragmentation paths discussed above as well. In the case of IndAc3433 (2), both loss of azetidine or transamination would form intermediates that could give rise to fragments $\mathbf{a}_{\mathbf{4 3}}$ and $\mathbf{b}_{43}$ and thus to the observed signals at $\mathrm{m} / \mathrm{z}$ $129 / 112$ (see Figure 1b). In this case, the signal for the secondary parent ion $[\operatorname{IndAc343}+\mathrm{H}]^{+}(\mathrm{m} / \mathrm{z} 360)$ proposed for the stepwise pathway is not found in the spectrum, which is possibly due to the fact that this ion should decompose immediately after its formation to fragment $\mathbf{a}_{\mathbf{4 3}}$-and subsequently $\mathbf{b}_{43}$ through a favored five-membered transition structure as described above. For IndAc3343 (3), the doublet at $\mathrm{m} / \mathrm{z} 129 / 112$ can be the response to the fragments $\mathbf{a}_{43} / \mathbf{a}_{34}$ and $\mathbf{b}_{34}$ deriving either by direct substitutive fragmentation or by the more complex pathways discussed above (see Figure 1c). A doublet at $\mathrm{m} / \mathrm{z}$ $115 / 98$ for $\mathbf{a}_{33}$ and $\mathbf{b}_{33}$ is not observed for this compound, however, because $[\operatorname{IndAc33}+\mathrm{H}]^{+}(\mathrm{m} / \mathrm{z}$ 289), obtained by loss of terminal diaminoalkyl group, cannot undergo fragmentation to these ions similarly to a free triamine.

In the case of IndAc4333 (1), the explanation for the formation of the ions of type $\mathbf{a}_{43} / \mathbf{a}_{34}$ and $\mathbf{b}_{43}$ (see Figure 1a) is slightly different: Loss of any portion of the molecule from the amino terminus of the compound and also transaminations would never lead to an intermediary species that could form these fragments. However, loss of the acyl group and liberation of the protonated polyamine itself seems to be a favorable CID-reaction for Acyl4 derivatives [6]. The respective signal for $[\mathrm{PA} 4333+\mathrm{H}]^{+}$is in fact found at $\mathrm{m} / \mathrm{z} 260$ in the ESI-MS/MS of IndAc4333 (1), and decomposition of the free polyamine can give rise to the fragments $\mathbf{a}_{\mathbf{4}} / \mathbf{a}_{\mathbf{3 4}}$ and $\mathbf{b}_{\mathbf{4 3}}$. The corresponding $[\mathrm{PA} 4333+\mathrm{H}]^{+}$and $[\mathrm{PA} 3433+\mathrm{H}]^{+}$signals for Compounds 2-4 are of low abundance only or not observed at all (see Figure 1a-d).

For Acyl3 derivatives, the alternative formation of 4H-5,6-dihydro-1,3-oxazine species, represented in the spectra by the strong signals at $m / z 215$, is the dominant reaction of the head portion [6].

\section{Conclusions}

We have shown that acylpolyamines can form some fragments in ESI-MS/MS that are composed of internal portions of the polyamine moieties of the sample molecules. From a mechanistic point of view, a fragmentation path involving a transamidation was excluded. Two alternative mechanisms-by transamination followed by fragmentation, or by two subsequent fragmentations-are possible and cannot be distinguished. Signals of such fragments could lead to misinterpretations if they were regarded to be derived from the end-portion of the compounds. Thus, for the definite characterization and structural identification of acylpolyamines, particularly those natural products of low abundance that lack synthetic references, such fragments are of little relevance. Of more relevance is the overall fragmentation pattern of the samples, which is dominated by the presence and location of 1,4-diaminobutane subunits. Such subunits allow some specific and predominant fragmentation reactions, occurring via five-membered transition structures. To deduce a specific sample structure on the basis of MS behavior, it is consequently necessary to judge the overall behavior of the considered compounds. In certain cases, the structural deduction might even not be feasible directly without the possibility to compare the sample compound with authentic samples.

\section{Acknowledgments}

The authors thank the Swiss National Science Foundation for their generous financial support and Professor Dr. M. Hesse for support of the project.

\section{References}

1. Schäfer, A.; Benz, H.; Fiedler, W.; Guggisberg, A.; Bienz, S.; Hesse, M. Polyamine Toxins from the Venom of Spiders and Wasps. Alkaloids 1994, 45, 1-125.

2. Bienz, S.; Detterbeck, R.; Ensch, C.; Guggisberg, A.; Häusermann, U.; Meisterhans, C.; Wendt, B.; Werner, C.; Hesse, M. Putrescine, Spermidine, Spermine, and Related Polyamine Alkaloids. Alkaloids 2002, 58, 83-338.

3. Itagaki, Y.; Nakajima, T. Acylpolyamines: Mass Spectrometric Analytical Methods for Araneidae Spider Acylpolyamines. J. Toxicol. Toxin Rev. 2000, 19, 23-52.

4. Chesnov, S.; Bigler, L.; Hesse, M. Detection and Characterization of Natural Polyamines by High-Performance Liquid Chromatography-Atmospheric Pressure Chemical Ionization (Electrospray Ionization) Mass Spectrometry. Eur. J. Mass Spectrom. 2002, 8, 1-16.

5. Chesnov, S.; Bigler, L.; Hesse, M. The Acylpolyamines from the Venom of the Spider Agelenopsis aperta. Helv. Chim. Acta 2001, 84, 2178-2197.

6. Manov, N.; Tzouros, M.; Chesnov, S.; Bigler, L.; Bienz, S. Solid-Phase Synthesis of Polyamine Spider Toxins and Correlation with the Natural Products by HPLC-MS/MS. Helv. Chim. Acta 2002, 85, 2827-2846.

7. Kramer, U.; Guggisberg, A.; Hesse, M.; Schmid, H. Transamidation Reactions. 2. The "Zip"-Reaction: A New Method for 
Ring Enlargement; Synthesis of 17- and 21-Membered Polyaminolactams. Angew. Chem. 1977, 89, 899-900.

8. Hu, W.; Reder, E.; Hesse, M. Neighboring-Group Participation in the Mass-Spectral Decomposition of 4-Hydroxycinnamoyl-Spermidines. Helv. Chim. Acta 1996, 79, 21372151.

9. Bigler, L.; Schnider, C. F.; Hu, W.; Hesse, M. ElectrosprayIonization Mass Spectrometry. Part 3. Acid-Catalyzed Isomerization of $N, N^{\prime}$-bis[(E)-3-(4-Hydroxyphenyl)prop-2enoyl]spermidines by the Zip Reaction. Helv. Chim. Acta 1996, 79, 2152-2163.

10. De Maaijer-Gielbert, J.; Gu, C.; Somogyi, A.; Wysocki, V. H.; Kistemaker, P. G.; Weeding, T. L. Surface-Induced Dissoci- ation of Singly and Multiply Protonated Polypropylenamine Dendrimers. J. Am. Soc. Mass Spectrom. 1999, 10, 414-422.

11. Zhu, C.; Jiang, Y.; Yang, X.; Zhao, Y. Electrospray Ionization Mass Spectra of Monoimidazole/Polyamine Conjugates. Rapid Commun. Mass Spectrom. 2002, 16, 2273-2277.

12. Manov, N.; Tzouros, M.; Bigler, L.; Bienz, S. Solid-Phase Synthesis of ${ }^{15} \mathrm{~N}$-Labeled Acylpentamines as Reference Compounds for MS/MS Investigation of Spider Toxins. Tetrahedron 2004, 60, 2387-2391.

13. Polce, M. J.; Ren, D.; Wesdemiotis, C. Dissociation of the Peptide Bond in Protonated Peptides. J. Mass Spectrom. 2000, 35, 1391-1398. 